\title{
$\$$ Research Square \\ Digital Web Archive Collection and Storage Model Based on Soft Computing and Edge-Driven System
}

\section{Lili Xie ( $\square$ liwenzhang8@126.com )}

NCUST-Affiliated Hospital

Jinbi Zhao

NCUST-Affiliated Hospital

Rui Sun

NCUST-Affiliated Hospital

\section{Research Article}

Keywords: Multimedia Technology, Intelligent Information Management System, Digital Storage, Soft Computing, Edge-Driven System

Posted Date: April 26th, 2021

DOI: https://doi.org/10.21203/rs.3.rs-436526/v1

License: (9) This work is licensed under a Creative Commons Attribution 4.0 International License. Read Full License 


\title{
Digital Web Archive Collection and Storage Model Based on Soft Computing and Edge-Driven System
}

\author{
Lili Xie ${ }^{1}$, Jinbi Zhaor, ${ }^{2}$ Rui Sun ${ }^{3}$
}

\author{
1. North China University of Science and Technology Affiliated \\ Hospital, Tangshan,Hebei, 063000, China \\ 2. North China University of Science and Technology Affiliated \\ Hospital, Tangshan,Hebei, 063000, China \\ 3. North China University of Science and Technology Affiliated \\ Hospital, Tangshan,Hebei, 063000, China \\ Corresponding author: Lili Xie
}

liwenzhang8@126.com

\begin{abstract}
With the continuous development of archives information network, it has become an important means to obtain archives information resources based on network means. At the same time, the number of users of network archives is increasing rapidly. It has become an urgent task to carry out the research on network archives users and do a good job in the management of network archives users. As the main body of archives utilization, archives users play a leading role in the content and direction of archives management and utilization. The informatization of archives management is not equivalent to computerization or networking, but a systematic project related to the whole educational reform and modernization. As the modern archives management has become a complex system, there are also multi-level division methods. The first level is divided into two subsystems: Archives entity management and archives information development, and each subsystem is further divided into several levels of small systems. Archives entity management is divided into collection, sorting, identification, storage, statistics and other work links. The whole file management system and its subsystems form feedback mechanism in operation. With the rapid development of computer technology and network technology, multimedia information system is penetrating into people's lives. Multimedia information system mainly includes three parts: central management system, terminal display system and network. Based on the multimedia information management system,
\end{abstract}


this paper constructs a digital network archives collection and storage model.

Keywords: Multimedia Technology; Intelligent Information Management System; Digital Storage; Soft Computing; Edge-Driven System

\section{Introduction}

With the strengthening of social archives security protection, people pay more and more attention to archives. As the main body of archives utilization, archives users play a leading role in the content and direction of archives management and utilization. The research on archives users is helpful for archives institutions to better understand users' needs and provide archives information services with high efficiency and high quality. With the rapid development of the Internet and the Internet, the network and the network of the public are becoming more and more important. At the same time, government departments, companies, scientific research institutions, etc. have built various forms of network platforms, and with the help of proprietary or public network platforms, more and more file information is provided, and archives resources can be consulted [1-4]. With the emergence of network technology, new changes have taken place in the information demand of archives users, the utilization and management methods of archives resources. The research of Archives Users under the network environment has attracted the attention of the archival circles. Some archival scholars and practical workers have begun to explore the influence of the network environment on the process and subject of archives utilization, and the research on archives users has become a hot spot again [5-8].

For a long time, the passivity of archives organizations has seriously hindered the users' requirements for high efficiency and high quality of information services in the network environment. The traditional way of providing information services is too simple to take into account the needs of the overwhelming majority of the people. Therefore, the Internet provides us with an open and real-time information retrieval platform. More and more archives information users can obtain the required file information without the limitation of time and space. Therefore, under the background of national archives informatization, it is necessary for us to conduct a comprehensive research on the archives information service under the network environment, and understand the research status and development trend of archives information service [9-12]. The ultimate purpose of file management is to provide file information for social practice. The structure of file management system is set up according to this purpose. Each of them is essential and has a certain procedure. They form an organic whole and play their respective roles in order to realize the overall function of the file management system, and at the same time, they are interrelated and mutually restricted [13-16].

The significance of network document archives management system is as follows:

(1) Strengthen the whole process management requirements of archives. Archives management business covers the traditional archives plan management business, management conditions and innovation environment construction, as well as approval, audit business. In order to better management, we need to manage all these businesses through a set of systems, so we need to build an integrated management system.

(2) Strengthen the cooperation ability of archives management personnel. The archives plan involves different levels of archives management departments such as the document and archives management department, many different positions in different business departments of the document and archives department, the decision-making 
level, the management level and the executive level, and a large number of experts, intermediary institutions and archives units. Management system can effectively improve the efficiency of their collaborative work.

(3) Improve the interaction between archives office and enterprises and institutions. Enterprises are the main archivists of science and technology plan, experts play an important role in the process of science and technology plan management, and recommendation units and intermediary organizations are important participants in science and technology management. However, there is no effective means to interact with them seamlessly. Through the construction of this management system, online communication and directional notice can be sent to specific groups. These means can effectively strengthen the interaction between government and enterprises.

(4) Realize the sharing of archives resources. Due to the division of work, different archives resources are managed by different people. There are a lot of important files and information management systems in it. Through an effective authorization mechanism, people with authority can view all relevant information.

(5) Establish the basis of daily management of archives. Through the construction of management system, more and more expert databases with higher quality can be established. It can establish a standard project database which is convenient for decision analysis in the future. The whole process of planning, archives, acceptance, grouping, review, approval, publicity, project establishment, tracking and supervision, and final acceptance are completed through the management system. The system will automatically remind the relevant personnel of the work to be done at the critical moment, which can be convenient for directional release of announcements. The system can flexibly select the relevant list and send e-mail, fax and SMS in groups.

(6) Strengthen project supervision. Due to the long project cycle, the large number of projects to be supervised, and the different emphasis to be supervised in different stages, the project supervision work is difficult to achieve. The management system can set the supervision time and automatically remind the relevant personnel for inspection. Automatically count and screen the list of enterprises that do not meet the regulatory requirements. This provides a powerful weapon for the project supervision of archives management personnel.

Multimedia technology is a new computer technology developed in 1980s. The development of computer multimedia technology is a process of gradual accumulation. In the 1950s, the processing and application of computer is numerical calculation, which takes digital media as its processing object. In the 1960s, it was extended to data processing. It was a combination of computer and character processing technology and text processing technology. It took digital and character media as processing objects. Then, the combination of computer, graphics and mathematics led to the computer-aided design technology mainly dealing with graphic media. With the combination of computer and image processing technology, computer image processing technology based on processing image media is produced. With the combination of computer and speech processing technology, the computer speech processing technology mainly dealing with voice media is produced. The multimedia technology developed in 1980 s is not only a comprehensive integration of the above technologies, but also a close combination of computer and image processing technology. As a result, a multi-media technology is formed, which takes the computer as the tool and sets the media such as picture, text, sound, dynamic and static images as the comprehensive processing object. The emergence of multimedia technology has formed a new technology content of computer, providing a new application environment for users [17-20].

Nowadays, multimedia information publishing system provides accurate and realtime public information for the public. Government departments, schools, enterprises and even military forces are widely using multimedia information release system [21-24]. It 
can be seen that the release of information through multimedia has greatly enriched the channels and presentation of information, increased the comprehensiveness and timeliness of information, and achieved good social benefits.

\section{Literature Review}

The research on archives users in China can be traced back to the theory of archives utilization in 1960s. The development of archives utilization theory involves the problem of archives users. At the end of 1980s, archival user research has gradually developed in our country, but the attention to user needs research is gradually developing, and there is no theoretical system like that of the shape image information user research. At the end of the 20th century, with the integration of computer technology into the field of archives service, the trend of digitalization of archives information resources is obvious, which has an impact on the research of archives users, and the research of network archives users has begun to attract attention. Guo Xiaoyun thinks that the paid archives information service implemented by archives institutions under the network environment is the extension and development of the traditional paid archives service under the market economy, which is normal and appropriate. At the same time, she also proposed that the fees charged for the online archives information services include the fees for the traditional archives paid services, as well as the new fees for providing services under the network environment, such as the digital fees generated through the network. Jin Fan believes that the formation of archives, the nature of archival work and the realization law of archival value determine and affect the research and development of Archival Information Service under the network environment to a certain extent. Under the network environment, the archives information service is becoming more and more socialized, and the most typical performance is the industrialization of the whole information service. The industrialization of network archives information service will also be an important part of information service industrialization. Archives information service is an important part of national public service, which should play an active and important role in scientific research and social production practice.

In recent years, driven by the strong demand for office informatization, Chinese government departments and large and medium-sized enterprises have rapidly promoted their own information construction. VCD was first designed in China and has been widely used. The Institute of acoustics of Chinese Academy of Sciences has made outstanding contributions to the design and industrialization of MPEG-1 decoding equipment. The MPEG-2 decoding equipment jointly completed by Harbin Institute of technology and the national intelligent computer research and development center of the Institute of computing, Chinese Academy of Sciences, has been successfully applied to DVD and digital satellite TV receivers, and has entered the industrialization stage [2528]. In the mid-1980s, the concept of multimedia was introduced into China, and some methods of multimedia technology were gradually realized. In the 1990s, multimedia technology began to attract attention and development. The second stage is the standardization stage. In this century, multimedia information publishing technology has been developed rapidly because of its good visual interface. At this time, large domestic enterprises and institutions and many government departments have built multimedia information publishing system based on client server system. The advantage of client server system is that the information resources are saved on the server, and the flexibility of multimedia information is realized. The application system based on the client server system also has some shortcomings, such as strong dependence on the operating system and poor scalability, which can not meet the growing needs of users. At this time, the third stage of the vigorous development of multimedia publishing technology came into 
being, and the multimedia information publishing system based on network platform came into being. The system can manage and broadcast multimedia information in real time.

\section{The Proposed Methodology}

\subsection{Demand Analysis of Multimedia Information System}

According to the software requirements specification of the system, the functions of the multimedia information publishing system are as follows: users can log in and operate on the web page. The multimedia system manages and sets up various types of multimedia materials, and sends the information to the multimedia terminal for publishing or playing. Multimedia materials include electronic documents, pictures, recording, video, flash, etc. In addition, the system has the function of managing these multimedia terminals.

The system consists of three parts

Server: it provides collaborative management, data storage and distribution functions of all terminals in the whole network, and provides address mapping between management terminal and playback terminal.

Management terminal: provides basic management and program production of data such as material, program, broadcast table, broadcast terminal and user, as well as remote control of broadcast terminal.

Broadcast terminal: it provides the automatic play of programs and play lists and the emergency broadcast of inserted contents, and accepts the remote control of the management terminal. At the same time, it sends heartbeat data to the server regularly to maintain online status.

The components of multimedia information system are shown in Figure 1.

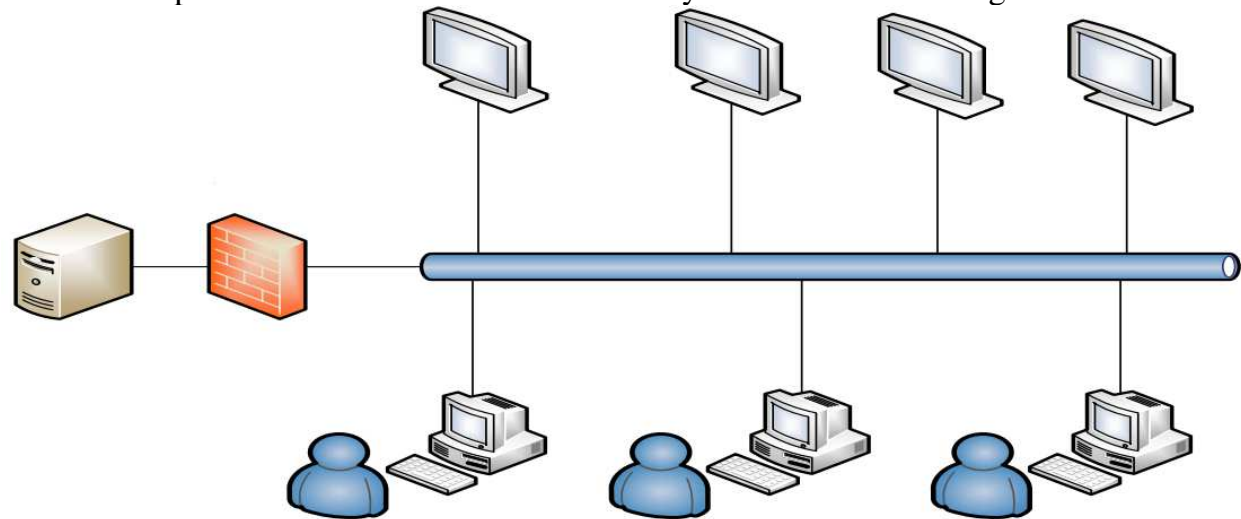

Figure 1. Multimedia information system component diagram

The main functions of the management terminal are to manage the multimedia information resources on the server, edit the multimedia resources to be played, and set the playing order and start time of the playing files on the playing end. It mainly realizes the functions of five parts: authority management, resource management, program arrangement, broadcast table editing and terminal management. Specific functional requirements are described below.

(1) Authority management. The user login interface is provided on the home page of the system, after which the user can use the system. The user permissions of the system are divided into ordinary users and administrator users. This system is not open to the public registration, only the administrator user has the authority to register new users. 
Administrators can also delete the ordinary users, the system to determine the rights of resources.

(2) Resource management. Users can upload local multimedia resources to the server through the resource management function, and can query the resources stored on the server. You can download the multimedia resources in the server to the local, or you can delete the resources in the server.

(3) Programming. Users can edit the multimedia resources on the server to produce the broadcast program through the program arrangement function.

(4) Broadcast table editing. Users can edit the prepared programs through the playlist editing function, and combine one or more programs into a playlist by adding playing properties to the programs.

(5) Terminal management. Terminal refers to the playing terminal. Users can control and manage the playing terminal through terminal management.

For the current system architecture in the software development field, there are mainly two software architectures to choose from: the first one is client/server mode $(\mathrm{C} / \mathrm{S})$; The second is the Web-based distributed object computing model, known as the $\mathrm{B} / \mathrm{S}$ model.

Distributed on client and server. In $\mathrm{C} / \mathrm{S}$ architecture, the server mainly provides data management, data sharing, data and system maintenance, concurrency control and other functions, while the client program mainly completes the specific business of the user. The user issues instructions to the server through the client, and the server extracts corresponding data according to the instructions received and sends them to the client. Then the client performs maintenance operations such as adding, modifying and deleting the received data, or sends the running results back to the server. When there is a large amount of data interaction between the two parties, it is likely to cause network congestion. $\mathrm{C} / \mathrm{S}$ mode is usually used to reduce network traffic by processing data in the background database and then sending the processing results to the client. This avoids sending large amounts of data to the client for processing [29-32].

$\mathrm{C} / \mathrm{S}$ architecture mainly has the following characteristics:

Strong interactivity. In $\mathrm{C} / \mathrm{S}$ architecture, the client is a complete set of applications, with very powerful functions in data processing, application interaction and other aspects. In $\mathrm{C} / \mathrm{S}$ architecture, the development of user interface is relatively simple and fast. There is no need to define interface control style or adopt layout technology to control the interface, so the clear layout of system pages can be easily realized.

Provides a more secure access mode. Because $\mathrm{C} / \mathrm{S}$ architecture is a point-to-point structure pattern in which the server and the client are paired together, it adopts a network protocol with good security, so it has high security.

It has fast execution speed. Because the $\mathrm{C} / \mathrm{S}$ architecture's business logic is processed on the client side, the results are then submitted to the server. Client response speed is fast, can effectively improve the execution speed of the system.

In $\mathrm{B} / \mathrm{S}$ architecture, the user's working interface is the web page of the browser, a small amount of data logic is processed in the front end, and the main data logic is processed in the server side. $\mathrm{B} / \mathrm{S}$ architecture is a network architecture pattern after the rise of the Internet, and the most important application software of the client is Web browser. This pattern makes the client more unified, and it centralizes the core functions of the system to the server. This makes it easier to develop, maintain, and use the system.

$\mathrm{B} / \mathrm{S}$ architecture mainly has the following characteristics:

Compatible with various operating systems and software platforms. The client can be realized through a common browser, which can save a lot of hard disk space and memory of the client, and the installation process is very simple.

System maintenance, expansion and upgrading is simple and convenient. Since most $\mathrm{B} / \mathrm{S}$ clients are browsers, there is little need to do any maintenance on them. Maintenance 
of the administrative server is generally required. When the system is upgraded, only the server side is upgraded. It is relatively easy to upgrade and maintain the system, which can save a lot of manpower, material resources, time and cost.

The function design of multimedia information release system adopts the scheme of module subdivision. In the overall function, the system is designed as database module, management terminal module, playing terminal module. Management terminal module includes login management module, user authority module, resource management module, programming module, playlist editing module, client management module, etc. The playing terminal module includes the playing interface module, interactive module, resource download module and so on.

\subsection{ASP.NET based System Development}

ASP.NET provides a component-based approach to design, develop, assemble, and deploy enterprise applications. Its goal is to provide a platform-independent, multi-user enterprise-level security development platform for B/ s-based servers. Figure 2 shows the three-tier architecture of ASP.NET technology.

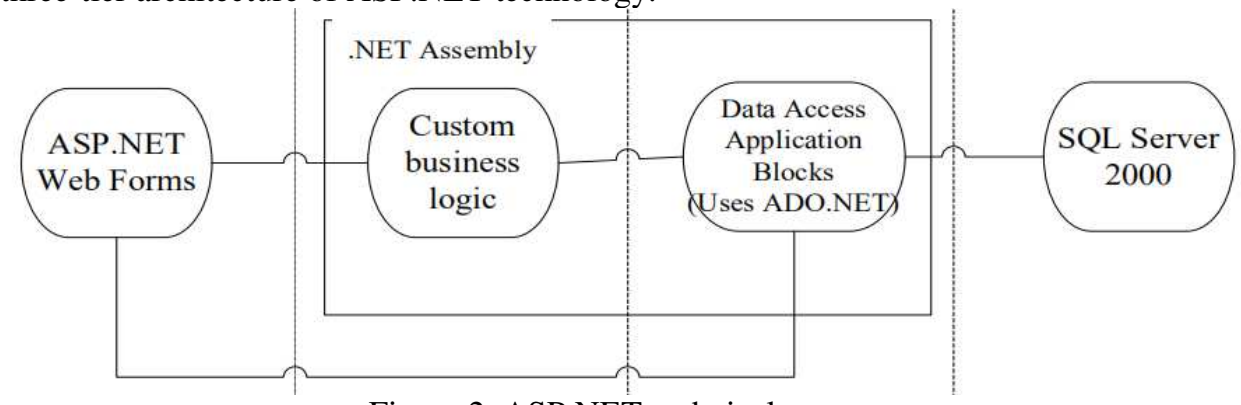

Figure 2. ASP.NET technical system

For software system, database technology is the advanced stage of data management. Database technology is to use the idea of data storage to organize and standardize the data model, and store the canonical data structure in memory to form a regular data set. A database provides application-level application services to a specific organizational structure in an optimized manner. The purpose of a data warehouse is to provide support for decision making through the analysis of historical information and subject-oriented information in the database. Traditional business systems make it difficult to get information about the state of the business. Data warehouse is a part of enterprise decision support.

Before the design and development of software engineering project, it is necessary to establish the model. The UML modeling tool used in this article is the Rational Rose environment, and its working interface is shown in Figure 3. 


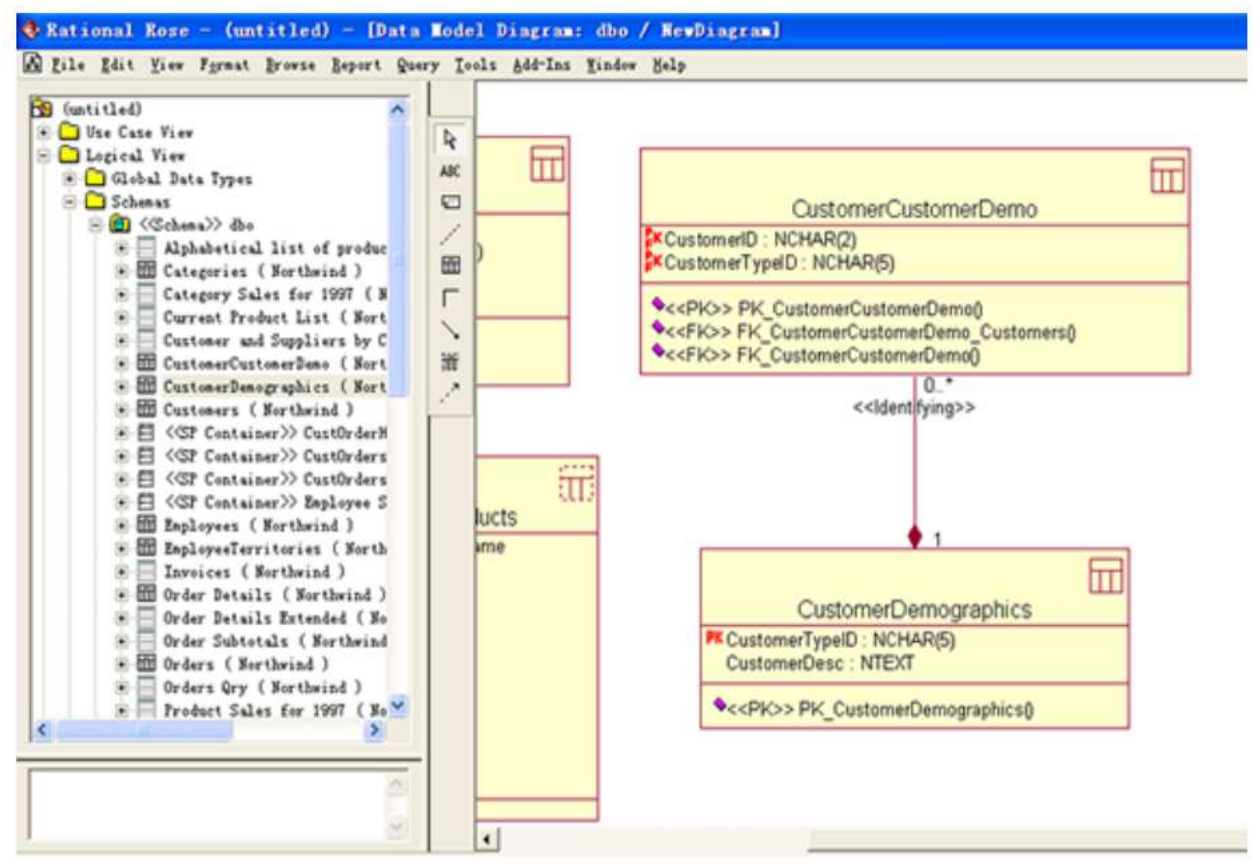

Figure 3. The working interface of Rational Rose software

The Rational Rose tool simplifies, extracts, and refines the architectural and functional modeling of software systems to reduce unnecessary design costs. Using the development tool to build UML model can effectively meet the designer's design requirements and extension requirements in the software development cycle

Asynchronous JavaScript and XML (AJAX) is a Web development technique for creating interactive web applications. In AJAX, XMLHTTPRequest is a core technology that provides a way for JAVASCRIPT scripts on a page to communicate with the server through these scripts. The JAVASCRIPT within the page can interact with the server without refreshing the page, that is, the page can either fetch data from the server or submit data to the server. Compared to traditional WEB technologies, AJAX can maintain data without updating the entire page. This allows Web applications to respond to user actions more quickly and avoids sending messages over the network that have not changed.

ASP.NETAJAX includes the server-side part and the client-side part, among which the server-side part of ASP.NETAJAX provides five server controls:

ScriptManager control: The script that manages the page.

ScriptManagerProxy control: The script for managing the page.

The UpdatePanel control: Together with the ScriptManager control, provides a refreshing Web environment.

Timer Control: Known as a Timer, it can trigger user-defined actions on time.

UpdateProgress control: Displays the progress of an entire or partial page.

The code created by the client is as follows:

xmlhttp_request $=$ new ActiveXObject( $" M s x m l 2 . X M L H T T P .3 .0 ") ; / / 3.0$

xmlhttp_request $=$ new ActiveXObject("Msxml2.XMLHTTP");

xmlhttp_request $=$ new ActiveXObject("Microsoft.XMLHTTP");

Once you have created the XMLHttpRequest, you can call the open() and send() methods of the HTTP request class, as follows: 
xmlhttp_request.open ('GET', URL, true);

xmlhttp_request.send (null);

\subsection{Construction of Multimedia Information Technology Management System}

The information of multimedia information resources usually comes from two aspects: one is the non-borrowing part, which is usually added by oneself; The other is usually information collected over the Internet or provided by others. AJAX is used extensively in the process of multimedia data uploading. When selecting a file, the system asynchronously retrieves the properties of the file and other related properties. This can be statistics background accurate statistics of some search information, and do not require user participation. This approach can improve the accuracy of information, and improve the efficiency of adding information and user's sense of experience.

The code for the unrefreshed upload method is as follows:

protected void DatePicker1_SelectionChanged(object sender, EventArgs e)

\{

Label1. Text $=$ DatePicker1. DateValue. ToShortDateString();

\}

protected void Button1_Click(object sender, EventArgs e)

\{

if (FileUpload1 . HasFile)

$\{$ Label1. Text $=$ FileUpload1. FileName; $\}$

\}

private void PreSendRequestHeadersHandler(object sender, EventArgs e)

\{

HttpApplication application $=($ HttpApplication $)$ sender $;$

HttpResponse response = application. Response;

if (response. StatusCode $==302 \& \&$

AjaxFileUploadUtility . IsInIFrameAsyncPostBack(application . Request . Params))

\{

string redirectLocation $=$ response. RedirectLocation;

List $\langle$ HttpCookie $>$ cookies $=$ new List $<$ HttpCookie $>($ response. Cookies . Count);

for (int $i=0 ; i<$ response. Cookies . Count; $i++$ )

\{

cookies . Add(response. Cookies[i]);

\}

response. ClearContent();

response. ClearHeaders(); 
for (int $i=0 ; i<$ cookies. Count $; i++$ )

\{

response. AppendCookie(cookies[i]);

\}

response. Cache. SetCacheability(HttpCacheability . NoCache);

response. ContentType = "text/plain";

AjaxFileUploadUtility . WriteScriptBlock(response, true)

StringBuilder $s b=$ new StringBuilder();

TextWriter writer $=$ new StringWriter $(s b)$;

AjaxFileUploadUtility . EncodeString(writer, "pageRedirect", String . Empty, redirectLocation);

response. Write(sb . Replace("*/", "*//*"). ToString());

AjaxFileUploadUtility . WriteScriptBlock(response, false);

response. End();

\}

\}

public void Dispose() \{\}

)

<asp:UpdatePanel ID="UpdatePanell" runat="server"

UpdateMode="conditional" $>$

$<$ Triggers $>$

<asp:PostBackTrigger ControlID="Button1" />

$</$ Triggers $>$

$<$ ContentTemplate>

<ews:DatePicker ID= "DatePickerl" runat="server" UsingUpdatePanel="True" OnSelectionChanged="DatePicker1_SelectionChanged" |\rangle$\langle b r /\rangle$

<asp:Label ID="Label1" runat="server" $\rangle\langle/$ asp:Label $\rangle\langle$ br $/\rangle\langle$ br $/\rangle$

<asp:FileUpload ID="FileUpload1" runat="server" />

<asp:Button ID="Button1" runat="server"

Text="Upload" OnClick="Button1_Click" />

$</$ ContentTemplate $>$

$</ a s p: U p d a t e P a n e l>$

$</$ asp:UpdatePanel>

Because the file is very big, the background USES compressed file to upload, which can improve the speed of uploading. 


\subsection{Design of Network Archive Database}

The database should have a logical structure that describes the data name, type, storage location, length, and other related parameters. The design of database should support the basic application and function of multimedia information release system. The rationality and security of database design play a vital role in the system. The database must meet the following requirements:

Real-time: Fast access to data to meet the requirements of real-time functions.

Flexibility: Multiple ways of accessing databases should be provided, requiring at least one to be intuitive and easy to access.

Recoverability: the database system is required to quickly recover to the state before the failure after the system failure disappears.

Integrity: In all cases, the integrity of the data in the database should be guaranteed.

Multimedia information system is a relational database application system. All information processed by the system is stored in the database. In the development of database application system, the structure design of database is a very important problem. The multimedia information system database is implemented by SQL Server database, which is mainly composed of user data, program data, broadcast table data and resource data. In order to avoid reading data that does not exist, the data in all tables is not allowed to be null.

In an efficient multimedia storage server, the issues associated with a storage manager include:

(1) A variety of magnetic and optical storage devices are used to meet the needs of different multimedia applications. These storage devices can have different properties, and they will be modeled uniformly and integrated into a single multimedia storage manager.

(2) Multimedia data requires a large storage space. In some application environments, the third and multiple storage layers must be used. In terms of multimedia application requirements, it is very important to make unified and effective integration for multi-level storage.

(3) The real-time retrieval performance of some multimedia objects requires quite a lot of running time. It is necessary to develop appropriate scheduling and resource allocation algorithms to achieve good performance in environments where delay sensitive data and non-delay sensitive data are mixed.

(4) Multimedia data is generally characterized by large volume and real-time retrieval. Therefore, it is necessary to effectively use the parallel mechanism of storage and processing in such systems.

(5) Many important multimedia information system applications are actually distributed. In order to ensure the system has a good performance of multimedia data retrieval, it is necessary to have the support of distributed system.

In a database environment, a combination of collection-oriented lookup and cruiseoriented browsing techniques is commonly used. Hypertext - and hypermedia-oriented systems use linked lists to cruise the database to find relevant concepts. Techniques such as keyword lists and relevance feedback are also used to create user profiles for effective query definitions and refinement. Typically, information is received through a variety of information sources and stored in an internal representation that facilitates addressable access, efficient display, and information extraction of content. The purpose of information extraction and display is to extract the associated information from the repository and present it to the user in the most appropriate form. This processing often involves the transformation of information.

Distributed hierarchical storage management functions include migration and retrieval tasks: 
1. Migration is the process of moving data from a client to a migration library, which is a remote mass storage device.

2. Retrieval is the movement of data from a remote storage device back to the original location of the client.

Distributed hierarchical storage management moves data between migrating clients and migrating storage servers. This migration is managed by a distributed hierarchical storage manager. A migration client is any computer on the network that contains data to be migrated. A migration server is a computer that provides migration services on the network. Migrating storage areas on the migration server can be disk, tape, or CD-ROM storage media.

\subsection{Design of Multimedia Information System Storage Management}

Multimedia data requires a lot of storage capacity: there are already multiple disks for archiving multimedia data. They are inexpensive, have large storage capacity, and have fast random access speed. Several other performance optimizations must be made to achieve the performance levels required for future VLBS. These optimizations include leveraging third-level storage, parallel mechanisms, hypercache, migration algorithms, scheduling, and query optimization, among others.

Multimedia storage managers should support scalability and storage transparency. In cases where multimedia data can take up a large amount of storage space, the system should be able to provide a variety of operations using multimedia data. .

In a multimedia information retrieval environment, a large number of objects meet a query requirement, but users do not all use them, because all they need is a subset of the data. At this point, it is more economical not to transfer these large objects to main memory until the query request is very precise.

Because of the data transfer time, the retrieval of large objects may be delayed. The system should try to reduce this delay by using the parallel mechanism of secondary storage. Different multimedia data types have different feature requirements, so it is necessary to use appropriate data layout algorithms for different data types.

The storage manager should be able to efficiently and transparently handle multiple storage layers. Main storage is the first storage layer, which is fast and growing in capacity and can be used to hold certain active parts of the database. A typical secondary storage layer consists of disks and optical disks, also known as secondary storage, which are used to store multimedia data with relatively high access probabilities. The third level of storage is used to store data that is not frequently accessed. Each disc has a number of read-write devices associated with it, and in larger configurations there may be more than one disc. The third storage layer has great capacity and can meet the space requirement of multimedia database application, but it is very slow.

With the development of multimedia technology, the speed of auxiliary storage device and the transmission speed of communication channel have been greatly improved. Fiber optic transmission rates can reach hundreds of megabits per second. However, the past practice shows that users will soon consume all kinds of additional resources they can get. Therefore, it is necessary to research and develop algorithms to ensure good realtime transmission and synchronization performance of multimedia information. These algorithms should use the super cache memory on the network, and use multimedia data model to ensure data transmission and synchronization.

Under the existing conditions, the storage management of multimedia information system can be designed based on a loosely coupled architecture. In other words, database management system is used to manage index information and file logical address, while file manager is used to manage multimedia files. An integration module is responsible for integrating DBMS and multimedia file manager. In this way, we can use the existing 
multimedia file management technology and DBMS technology to manage the storage of multimedia information system.

In the retrieval process, the query interface provided by the storage management system first receives the user's retrieval request and the related information of the query target. Then, the retrieval module of storage management system queries the index information base according to the index information provided by the user, and displays all index records in the index information base that meet the query target information provided by the user. At this time, the multimedia object itself is not transmitted to the local. When the user selects the multimedia data object according to the index record information displayed by the system, the retrieval module of the system communicates with the index information base again to obtain the logical address of the multimedia object. The system automatically finds the retrieved multimedia object from the multimedia storage server according to the logical address, and transmits the object back to the client for browsing.

\section{Experiment and Verifications}

This paper constructs a network file acquisition and storage model based on multimedia resource management. In the multimedia resource management module, it mainly displays all the resource information and provides retrieval approaches. The file list page and the query page are shown in Figure 4:

\begin{tabular}{|c|c|c|c|c|c|c|c|c|c|}
\hline wid & whame & wurl & wsize & wext & wtime & wtype & waddname & wdesc & wauthor \\
\hline 3 & $a b c$ & $a b c$ & $a b c$ & $a b c$ & $a b c$ & $a b c$ & $a b c$ & $a b c$ & $a b c$ \\
\hline 1 & $a b c$ & $a b c$ & $a b c$ & $a b c$ & $a b c$ & $a b c$ & $a b c$ & $a b c$ & $a b c$ \\
\hline 2 & $a b c$ & $a b c$ & $a b c$ & $\mathrm{abc}$ & $a b c$ & $a b c$ & $a b c$ & $a b c$ & $a b c$ \\
\hline 3 & $a b c$ & $a b c$ & $a b c$ & $a b c$ & $a b c$ & $a b c$ & $a b c$ & $a b c$ & $a b c$ \\
\hline 4 & $a b c$ & $a b c$ & $a b c$ & $a b c$ & $a b c$ & $a b c$ & $a b c$ & $a b c$ & $a b c$ \\
\hline 5 & $a b c$ & $a b c$ & $a b c$ & $a b c$ & $a b c$ & $a b c$ & $a b c$ & $a b c$ & $a b c$ \\
\hline 6 & $\mathrm{abc}$ & $a b c$ & $a b c$ & $a b c$ & $a b c$ & $a b c$ & $a b c$ & $a b c$ & $\mathrm{abc}$ \\
\hline$?$ & $\mathrm{abc}$ & $a b c$ & $a b c$ & $a b c$ & $a b c$ & $a b c$ & $a b c$ & $a b c$ & $\mathrm{abc}$ \\
\hline 8 & $\mathrm{abc}$ & $\mathrm{abc}$ & $a b c$ & $a b c$ & $a b c$ & $a b c$ & $a b c$ & $a b c$ & $a b c$ \\
\hline 9 & $a b c$ & $a b c$ & $a b c$ & $a b c$ & $a b c$ & $a b c$ & $a b c$ & $a b c$ & $a b c$ \\
\hline 10 & $\mathrm{abc}$ & $a b c$ & $a b c$ & $a b c$ & $a b c$ & $a b c$ & $a b c$ & $a b c$ & $a b c$ \\
\hline 11 & $a b c$ & $a b c$ & $a b c$ & $a b c$ & $\mathrm{abc}$ & $a b c$ & $\mathrm{abc}$ & $a b c$ & $\mathrm{abc}$ \\
\hline 12 & $\mathrm{abc}$ & $a b c$ & $a b c$ & $\mathrm{sbc}$ & $a b c$ & $a b c$ & $a b c$ & $a b c$ & $a b c$ \\
\hline 13 & $a b c$ & $a b c$ & $a b c$ & $a b c$ & $a b c$ & $a b c$ & $a b c$ & $a b c$ & $a b c$ \\
\hline 14 & $\mathrm{abc}$ & abc & $a b c$ & $a b c$ & $a b c$ & $a b c$ & $a b c$ & $a b c$ & $a b c$ \\
\hline 15 & $a b c$ & $a b c$ & $a b c$ & $a b c$ & $a b c$ & $a b c$ & $a b c$ & $a b c$ & $\mathrm{abc}$ \\
\hline 16 & $\mathrm{abc}$ & $a b c$ & $a b c$ & $a b c$ & $a b c$ & $a b c$ & $a b c$ & $a b c$ & $a b c$ \\
\hline 17 & $a b c$ & $a b c$ & $a b c$ & $a b c$ & $a b c$ & $a b c$ & $a b c$ & $a b c$ & $a b c$ \\
\hline 18 & $a b c$ & $\mathrm{abc}$ & $a b c$ & $a b c$ & $a b c$ & $a b c$ & $a b c$ & $a b c$ & $a b c$ \\
\hline 19 & $\mathrm{abc}$ & $\mathrm{sbc}$ & $a b c$ & $a b c$ & $a b c$ & $a b c$ & $\mathrm{abc}$ & $a b c$ & $\mathrm{sbc}$ \\
\hline
\end{tabular}

Figure 4. File list page and query page

The integration tests are shown in Table 1 for example. This table mainly reflects the different expected results represented by different TC-ids.

Table 1. Integrate test cases

\begin{tabular}{|c|c|c|c|}
\hline \multirow[t]{2}{*}{ TC_ID } & \multirow{2}{*}{$\begin{array}{l}\text { Functional test } \\
\text { case number }\end{array}$} & Input/operation & \multirow{2}{*}{$\begin{array}{l}\text { The expected } \\
\text { results }\end{array}$} \\
\hline & & $\begin{array}{l}\text { The object } \\
\text { being saved }\end{array}$ & \\
\hline $\begin{array}{l}\text { TC-SIT-Bulid1- } \\
001\end{array}$ & N/A & FILES & $\begin{array}{l}\text { Store the image } \\
\text { data in the database }\end{array}$ \\
\hline $\begin{array}{l}\text { TC-SIT-Bulid1- } \\
002\end{array}$ & N/A & FILES & $\begin{array}{c}\text { Store the } \\
\text { document data in } \\
\text { the database }\end{array}$ \\
\hline
\end{tabular}




\begin{tabular}{|c|c|c|c|}
\hline $\begin{array}{c}\text { TC-SIT-Bulid1- } \\
003\end{array}$ & N/A & FILES & $\begin{array}{c}\text { Store audio } \\
\text { format data in a } \\
\text { database }\end{array}$ \\
\hline $\begin{array}{c}\text { TC-SIT-Bulid1- } \\
004\end{array}$ & N/A & FILES & $\begin{array}{c}\text { Store video } \\
\text { format data in a } \\
\text { database }\end{array}$ \\
\hline
\end{tabular}

The performance test of the system mainly includes the following indicators: the response time of the management terminal page, the response time of the connection between the management terminal and the playing terminal, the response time of the receiving data of the playback terminal, and the continuous running time of the playback terminal. The test results are as follows:

Table 2. The test results of page response time

\begin{tabular}{|c|c|c|c|c|c|}
\hline $\begin{array}{c}\text { Number } \\
\text { of users }\end{array}$ & 1 & 2 & 5 & 7 & 10 \\
\hline $\begin{array}{c}\text { Page } \\
\text { response } \\
\text { time }\end{array}$ & $1.2 \mathrm{~s}$ & $1.2 \mathrm{~s}$ & $1.2 \mathrm{~s}$ & $1.2 \mathrm{~s}$ & $1.3 \mathrm{~s}$ \\
\hline
\end{tabular}

Table 3. The test results of connection response time

\begin{tabular}{|r|c|c|c|c|c|}
\hline $\begin{array}{c}\text { Number } \\
\text { of terminals }\end{array}$ & 1 & 2 & 5 & 7 & 10 \\
\hline $\begin{array}{c}\text { Connect } \\
\text { ion response } \\
\text { time }\end{array}$ & $2 \mathrm{~s}$ & $2 \mathrm{~s}$ & $2.1 \mathrm{~s}$ & $2.1 \mathrm{~s}$ & $2.3 \mathrm{~s}$ \\
\hline
\end{tabular}

\begin{tabular}{|c|c|c|c|c|c|}
\hline \multicolumn{7}{c|}{ Table 4. The test results of play response time } \\
\hline $\begin{array}{c}\text { Number } \\
\text { of terminals }\end{array}$ & 1 & 2 & 5 & 7 & 10 \\
\hline $\begin{array}{c}\text { Play } \\
\text { response } \\
\text { time }\end{array}$ & $5.8 \mathrm{~s}$ & $5.8 \mathrm{~s}$ & $6.2 \mathrm{~s}$ & $6.3 \mathrm{~s}$ & $7.2 \mathrm{~s}$ \\
\hline
\end{tabular}

\begin{tabular}{|c|c|c|c|c|c|}
\hline \multicolumn{7}{|c|}{ Table 4. The test results of uptime } \\
\hline $\begin{array}{c}\text { Running } \\
\text { time }\end{array}$ & $1 \mathrm{~h}$ & $5 \mathrm{~h}$ & $8 \mathrm{~h}$ & $12 \mathrm{~h}$ & $24 \mathrm{~h}$ \\
\hline $\begin{array}{c}\text { Operatio } \\
\text { n results }\end{array}$ & $\begin{array}{c}\text { normal } \\
\text { operation }\end{array}$ & $\begin{array}{c}\text { normal } \\
\text { operation }\end{array}$ & $\begin{array}{c}\text { normal } \\
\text { operation }\end{array}$ & $\begin{array}{c}\text { normal } \\
\text { operation }\end{array}$ & $\begin{array}{c}\text { normal } \\
\text { operation }\end{array}$ \\
\hline
\end{tabular}

It can be seen that the response time increases with the number of users or terminals. But the time is within the acceptable range of users, which ensures the real-time of multimedia information release.

After the user has configured the basic user configuration and data configuration information in the login interface, click the "confirm download" button to download the data. If a user authentication error occurs, the server returns an error message. If the user is authenticated, the server will return the user's authorized data information to the client. The interface for server-side users to download files is shown in Figure 5. 


\section{d: IrrqDisRadarServer|Release WisRadarServer. eze}

INFO [3752] connect.ConnectThread(139)(3752)10:59:14 INFO [3568] connect. Connect Thead $(563\rangle\langle 3568\rangle 10: 59: 14$

INFO [3972] connect. ConnectThread(563)(3972) 16:59:14

INFO [3752] connect. Connect Thread $\langle 563\rangle\langle 3752\rangle 16: 59: 14$ 18

INFO [3568] connect.ConnectThread(282)(3568)10:59:14 Weruerdata $\backslash$ PARSER_Uo ice

INFO [2868] connect.Connect Thread (339)(2868)16:59:14 Serverdata $\backslash$ PARSER_UIDEO

INFO [3072] connect. Connect Thread 423$\rangle(3672\rangle 16: 59: 14$ INFO [3752] connect. ConnectThread $(563\rangle\langle 3752\rangle$ 10:59:14 -

INFO [3972] connect . ConnectThread(442)(3672)10:59:14 -

INFO [3752] connect.Connect Thread $(394\rangle\langle 3752\rangle 10: 59: 14$ Serverdata PARSER_PICTURE

INF0 [3972] connect . ConnectThread $(563)(3072) 10: 59: 14$ -

INF0 [3752 1 connect. ConnectThead (394) (3752)10:59:14 -Serverdata PRRSER_PICTURE

INFO [3972] connect . Connect Thread $\langle 537\rangle\langle 3372\rangle 10: 59: 14$ 21101624

Figure 5. The interface for server-side users to download files The upload file interface is shown in Figure 6. 
ci d: IrqDisRadarServer IRelease VDisRadarServer-exe

INF0 [2712] connect .Connect Thread(423)(2712)11:20:69 INFO [2736] connect . Connect Thread (563)(2736)11:20:09

INFO [252] connect. ConnectThread(394)(252)11:20:69 NHיq Serverdata VARSER_PICTURE

INFO [2712] connect.ConnectThread (442)〈2712)11:20:99

INF0 [3568] connect. ConnectThead(282)(3568)11:20:69 D: Urq Lerverdata $\backslash$ PARER_Voice

INFO [252] connect . ConnectThread(394) $\langle 252\rangle$ 11:20:69 Wrq \Serverdata VPARSER_PICTURE

INFO [2712] connect . Connect Thread $(563\rangle\langle 2712\rangle 11=20: 09$

INFO [2736] connect.ConnectThread(339)(2736)11:20:69 D: Mrq \Serverdata VARSER_UIDEO

INFO [252] connect . Connect Thread $\langle 394\rangle\langle 252\rangle 11: 20: 69-$ Wrq \Serverdat a PARSER_PICTURE

INFO [2712] connect . ConnectThread (537)(2712)11:20:09 20110921085645

INF0 [252] connect.ConnectThread(394)(252)11:20:09 NHY Serverdata PARSER_PICTURE

INF0 [252] connect .ConnectThread $(394\rangle\langle 252\rangle$ 11:20:09 Figure 6. The page of file upload

The interface of multimedia information publishing system is shown in Figure 7. 

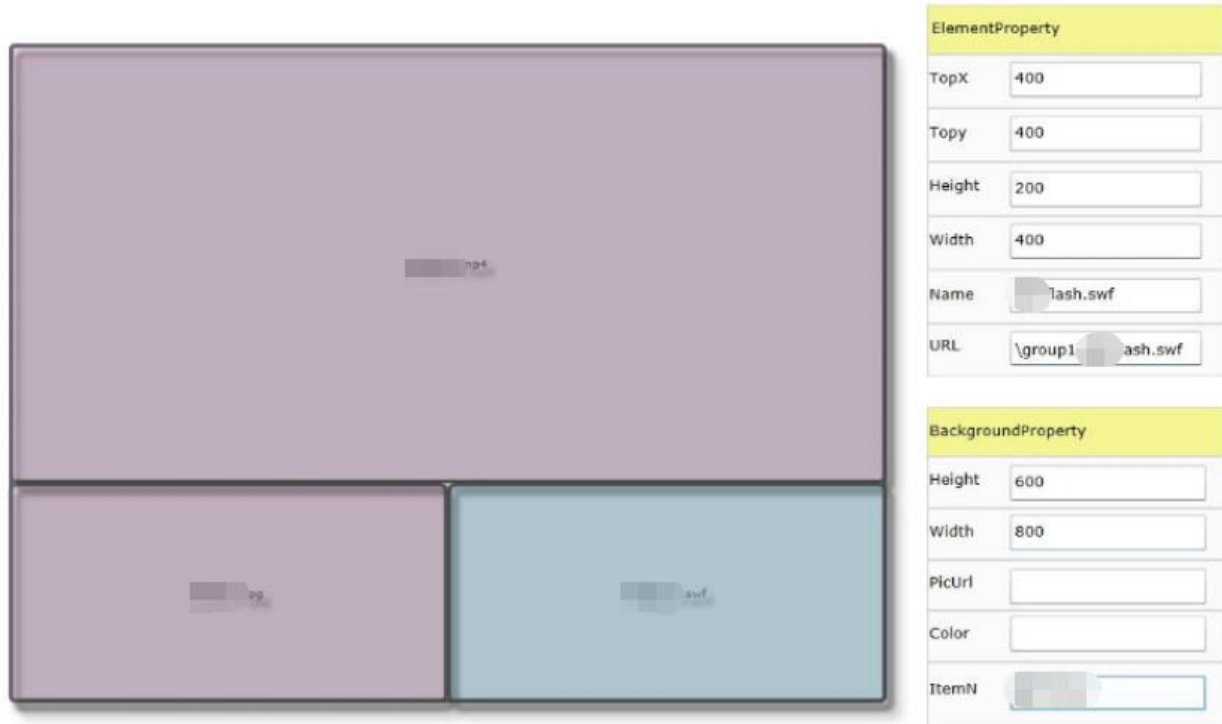

Figure 7. The page of item arrange

The effect of the system playback terminal is shown in Figure 8.
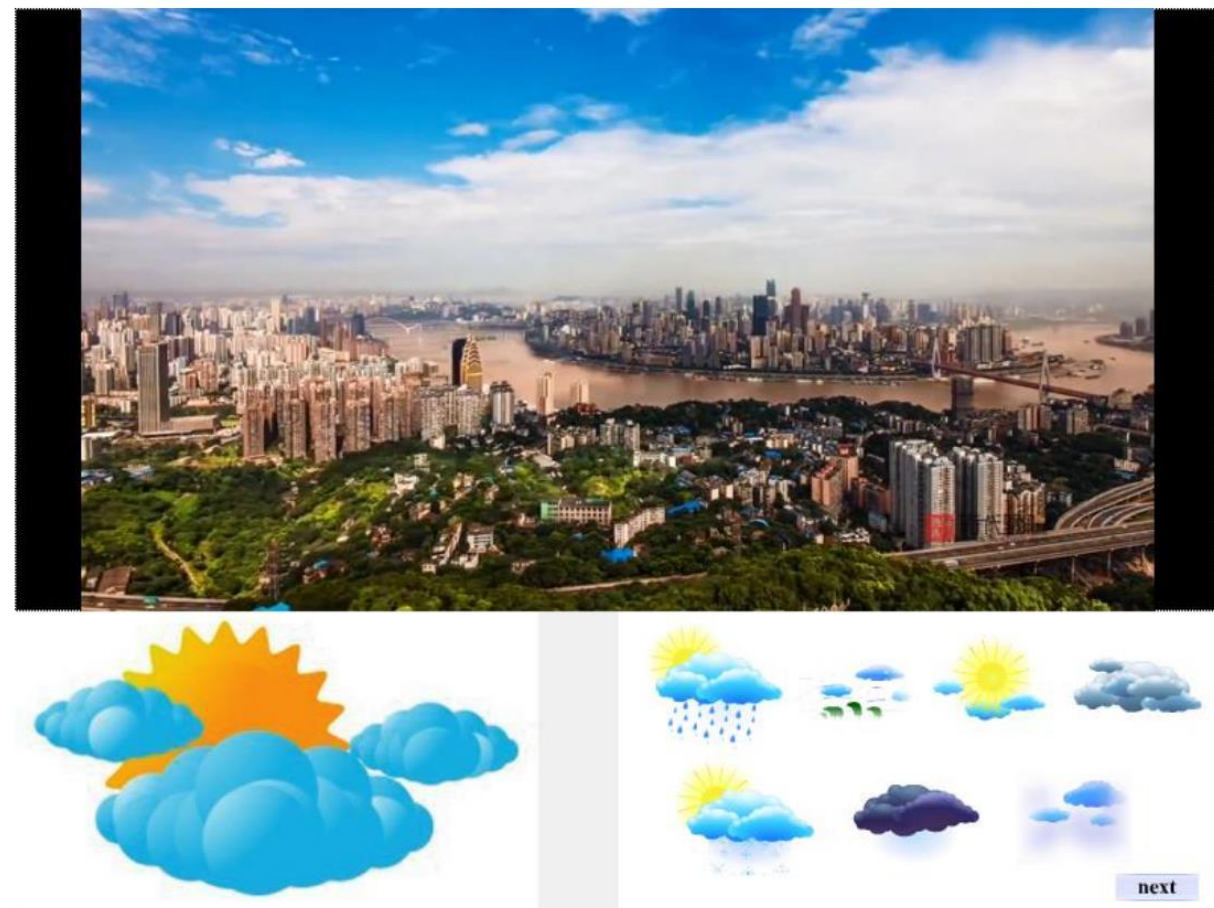

Figure 8. Play screen

It can be seen that the actual height and width of the player interface are $1680 * 1050$, and the playing resources are played on the interface according to scale. The file playing on the top of the interface is a video with a width and height of $1680 * 700$, the file playing on the bottom left of the interface is a picture with a width and height of $840 * 350$, and the file playing on the bottom right of the interface is flash with a width and height of 
$840 * 350$. On the player interface, you can play files with all three properties at the same time, and the same position as you would see on the edit panel when setting the program.

\section{Conclusion and Prospect}

Archival information resources are important information resources in our country. In particular, ensuring the security of archival information under the network environment is not only the subject of archival information work but also the important responsibility of the national information department. Once the problem of archival information security occurs under the network environment, the loss will be incalculable. In the process of developing archival information service under the network environment, we should do a good job in the construction of archival information database and deal with the relationship between network archival information service and archival information security. These problems affect the level and quality of archival information service in the network environment to different degrees. The initial stage of multimedia technology has achieved great success and shows a broad application prospect. But in designing some advanced multimedia systems, technical requirements far exceed what is available today. This shows that the further development of multimedia applications is expected to be driven by new breakthroughs in multimedia technology. At present, the research of distributed multimedia technology is one of the keys. With the continuous development of information and network technology, the traditional way of file management needs to be reformed. People have been used to the network to study and discuss, the network literature archive management to adapt to the development trend. Under the environment of ASP.NET development technology, this paper constructs a network file acquisition and storage system based on multimedia information technology. The document archives management system basically realizes the document archives management informationization business flow. The completion of this subject has certain practical significance and value in order to improve the automation level of literature archive management in Our country. The construction of document archive information service management is a dynamic development process, and the informatization of document archive management is an inevitable trend. The test results show that the interface of the multimedia information system proposed in this paper is beautiful and simple, convenient to use, low cost, and clear and smooth playback content. This paper realizes the goal of the system and all the requirements of the project, meets the application conditions of the project, and can be used widely.

Conflict of interest:

None

Author contribution:

The authors have the same contribution to the paper.

\section{References}

[1] Xue Sixin, Yang Yan, Huang Cunxun. Fundamentals of modern archives management [M]. Beijing: China Machine Press, 2006. 
[2] Meng Guangjun, Huo Guoqing, Roman, et al. Introduction to information resource management [M]. 2 edition. Beijing: Science Press, 2003.

[3] Huang Xiaobin. Development and management of network information resources [M]. Beijing: Tsinghua University Press, 2009.

[4] Zhang Zhaoyu. Theory and practice of current case informatization [M]. Beijing: China archives publishing house, 2007.

[5] Macleod, Schell. Archives management information system [M]. Beijing: Peking University Press, 2004.

[6] Li Xiaomao. Thinking on the development strategy of e-commerce of Chinese enterprises [J]. China soft science, 2000.

[7] Jing linbo. Strategic plan of e-commerce marketing [J]. China ecommerce,2001,21(3):56-72.

[8] Zhu Jianqiu. Data mart and data warehouse [J]. Chinese computer users, 2003.

[9] Du Dong. Information management course [M]. Beijing: Tsinghua University Press, 2002 (2): 21-23.

[10] Robert Tusler, An Overview of Project Risk Management, 29 July 1996.

[11] Preston G. Smith, Smith CMC, Guy M. Merritt, Managing Consulting Project Risk, 3 September 2002.

[12] Li Suhua, Zhang Yingping, Chen Guangzhen, Deng Yangling. Analysis of database management security measures [J]. Journal of Yellow River Water Conservancy Vocational and technical college, 2007119 (4): 121-123.

[13] Muriithi, N. \& Crawford, L. (2003). Approaches to project management in Africa: Implications for international development projects. International Journal of Project Management, 21(5), 309-319.

[14] Aladwani, A. M. (2002). IT Project uncertainty, planning and success: An empirical investigation from Kuwait. Information Technology and People. 15 (3), 210-226. 
M. Schoeberl. A Java processor architecture for embedded real-time systems. Journal of Systems Architecture, doi:10.1016/j.sysarc.2007.06.001, 2007.

[16] Warfield, John N., A Science of Generic Design, Managing Complexity Through System Design. lowa State University Press, AMES, IA,1994.

[17] Wang Chen, Du Shaoping, Li Guohui. Object relational database supporting technology for multimedia. Computer engineering and application, 2000,3.

[18] Ma Xiujun. Multimedia database and content retrieval. Peking University press,2007,7.

[19] Huang Zhijun, Zeng bin. Multimedia database technology. National Defense Industry Press, January 2005.

[20] Zhang Jun, Liu Wei, Li Guohui, et al. Development of multimedia database information retrieval application with Oracle. Science Press, 2005,11.

[21] Huang Yanling, Zhang Qi, Chen Chunxiao. Discussion on the construction of meteorological multimedia integrated information release system [C]. Abstract of 2011 Annual Meeting of meteorological communication and Information Technology Committee of China Meteorological Society and National Meteorological Information Center, 2011.

[22] Qi Xiao. Design and development of campus network multimedia information publishing system [D]. University of Electronic Science and technology, 2011.

[23] H. Ali, M. Hasan. Adaptive Time-Sharing Distribution Information Publication System[C]. International Symposium on Information Processing, 2010: 342-346.

[24] S. M. HOWELL,B. J. GALINAT. The Glenoid-Labral Socket: A Constrained Articular Surface[J]. Clinical orthopaedics and related research, 1989, 243: 122-125.

[25] Song Yujun. Data management in multimedia database. Gansu science and technology, March 2007. 
[26] Scot T . Campbell.The Role of Database Systems in the Management ofMultimedia Information. Application Service Definition and Protocol Specification, 2000.

[27] Dcot T . Campbell. The Managementof Database Systems in the Management ofMultimedia Informaiton. Application Service Definition and Protocol Specification, 2000.

[28] Ramaswamy S, RastogiR, Shin K. Efficient Algorithms Form Fining Outliers from Large Datasets $[C]$, Proceedings of the ACM SICMOD Conference,,2000,427-438.

[29] E. A. Fox. Advances in interactive digital multimedia systems[J]. Computer, 1991, 24(10): 9-21.

[30] Zhang Shouwei, song Wenai. Analysis of management information system based on C / S and B / S combination mode [J]. Information communication, 2007, 20 (6): 57-59.

[31] S. M. Lewandowski. Frameworks for component-based client/server computing[J]. Acm Computing Surveys, 1998, 30(1): 3-27.

[32] S. H. Plantz,J. Berezin, "Group publishing system," ed: Google Patents, 2000. 
Figures

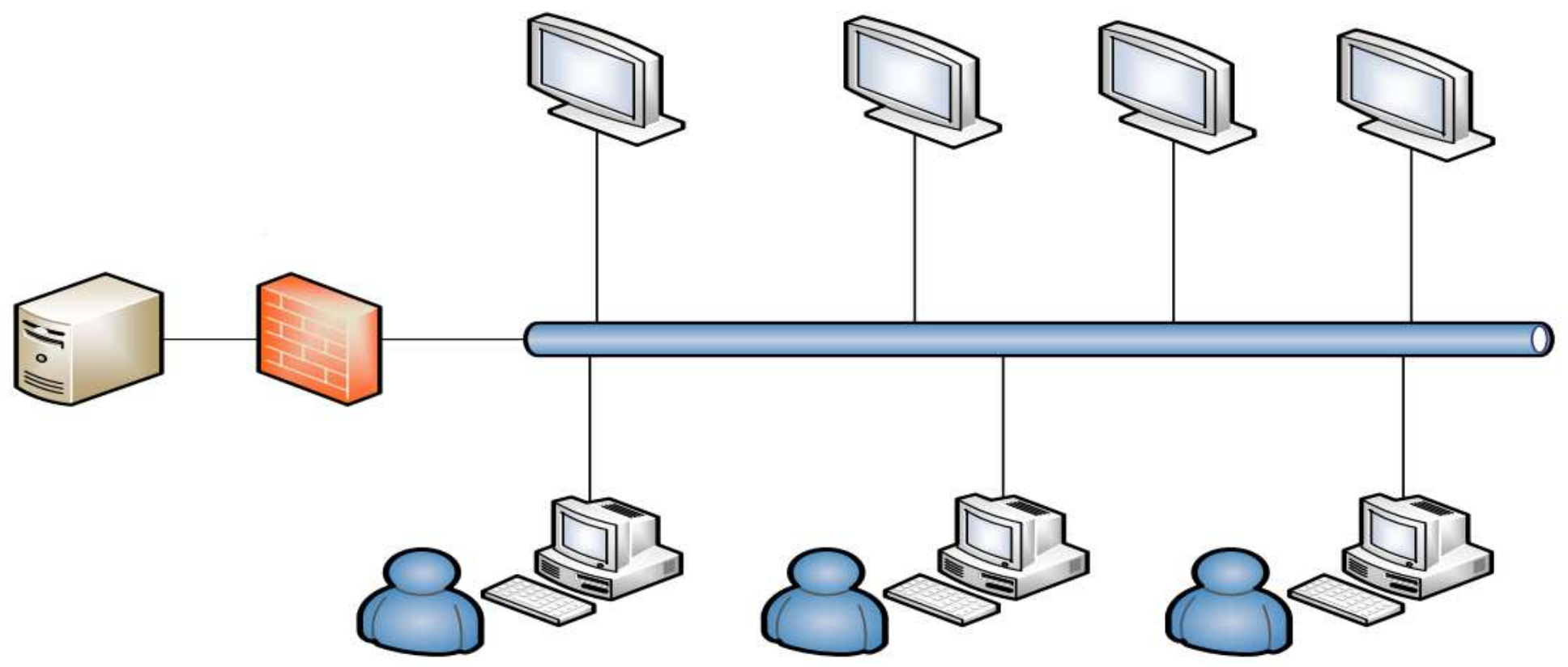

Figure 1

Multimedia information system component diagram

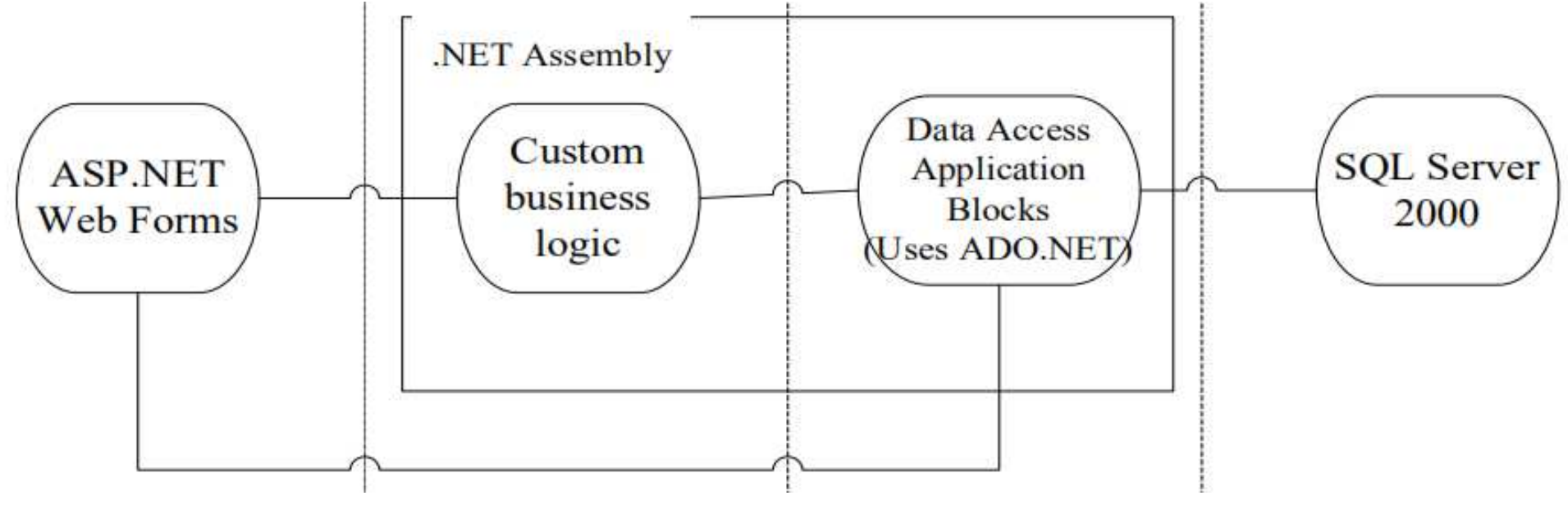

Figure 2

ASP.NET technical system 

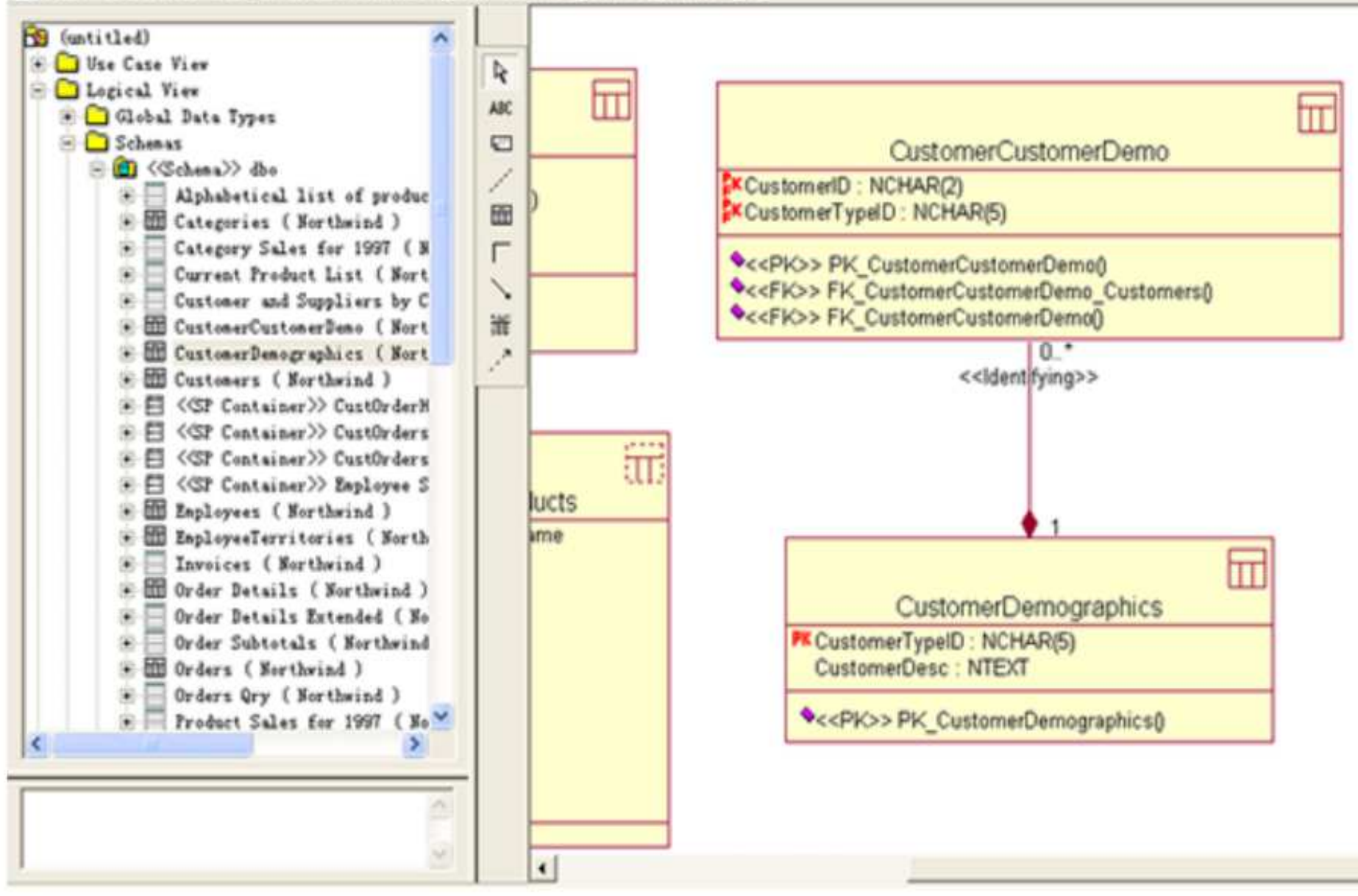

Figure 3

The working interface of Rational Rose software

\begin{tabular}{|c|c|c|c|c|c|c|c|c|c|}
\hline wid & wname & wurl & wsize & wext & wtime & wtype & waddname & $\underline{\text { wdesc }}$ & wauthor \\
\hline p & $a b c$ & $a b c$ & $a b c$ & $a b c$ & $a b c$ & $a b c$ & $a b c$ & $a b c$ & $a b c$ \\
\hline 1 & $a b c$ & $a b c$ & $a b c$ & $a b c$ & $a b c$ & $a b c$ & $a b c$ & $a b c$ & $a b c$ \\
\hline 2 & $a b c$ & $a b c$ & $a b c$ & $a b c$ & $a b c$ & $a b c$ & $a b c$ & $a b c$ & $a b c$ \\
\hline 3 & $a b c$ & $a b c$ & $a b c$ & $a b c$ & $a b c$ & $a b c$ & $a b c$ & $a b c$ & $a b c$ \\
\hline 4 & $a b c$ & $a b c$ & $a b c$ & $a b c$ & $a b c$ & $a b c$ & $a b c$ & $a b c$ & $a b c$ \\
\hline 5 & $a b c$ & $a b c$ & $a b c$ & $a b c$ & $a b c$ & $a b c$ & $a b c$ & $a b c$ & $a b c$ \\
\hline 6 & $a b c$ & $a b c$ & $a b c$ & $a b c$ & $a b c$ & $a b c$ & $a b c$ & $a b c$ & $a b c$ \\
\hline$\beta$ & $a b c$ & $a b c$ & $a b c$ & $a b c$ & $a b c$ & $a b c$ & $a b c$ & $a b c$ & $a b c$ \\
\hline 8 & $a b c$ & $a b c$ & $a b c$ & $a b c$ & $a b c$ & $a b c$ & $a b c$ & $a b c$ & $a b c$ \\
\hline 9 & $a b c$ & $a b c$ & $a b c$ & $a b c$ & $a b c$ & $a b c$ & $a b c$ & $a b c$ & $a b c$ \\
\hline 10 & $a b c$ & $a b c$ & $a b c$ & $a b c$ & $a b c$ & $a b c$ & $a b c$ & $a b c$ & $a b c$ \\
\hline 11 & $a b c$ & $a b c$ & $a b c$ & $a b c$ & $a b c$ & $a b c$ & $a b c$ & $a b c$ & $a b c$ \\
\hline 12 & $a b c$ & $a b c$ & $a b c$ & $a b c$ & $a b c$ & $a b c$ & $a b c$ & $a b c$ & $a b c$ \\
\hline 13 & $a b c$ & $a b c$ & $a b c$ & $a b c$ & $a b c$ & $a b c$ & $a b c$ & $a b c$ & $a b c$ \\
\hline 14 & $a b c$ & $a b c$ & $a b c$ & $a b c$ & $a b c$ & $a b c$ & $a b c$ & $a b c$ & $a b c$ \\
\hline 15 & $a b c$ & $a b c$ & $a b c$ & $a b c$ & $a b c$ & $a b c$ & $a b c$ & $a b c$ & $a b c$ \\
\hline 16 & $a b c$ & $a b c$ & $a b c$ & $a b c$ & $a b c$ & $a b c$ & $a b c$ & $a b c$ & $a b c$ \\
\hline 17 & $a b c$ & $a b c$ & $a b c$ & $a b c$ & $a b c$ & $a b c$ & $a b c$ & $a b c$ & $a b c$ \\
\hline 18 & $a b c$ & $a b c$ & $a b c$ & $a b c$ & $a b c$ & $a b c$ & $a b c$ & $a b c$ & $a b c$ \\
\hline 19 & $a b c$ & $a b c$ & $a b c$ & $a b c$ & $a b c$ & $a b c$ & $a b c$ & $a b c$ & $a b c$ \\
\hline 12 & & & & & & & & & \\
\hline
\end{tabular}


or d: lerqWisRadarServerlReleaseVDisRadarServer.eze

INFO [3752] connect.ConnectThread (139)(3752)10:59:14 INFO [3568] connect. ConnectThread $(563)(3568\rangle) 10: 59: 14$ -

INFO [3972] connect .ConnectThead $(563)(3972) 1.3: 59: 14$ -

INFO [3752] connect. ConnectThead $(563)(3752\rangle 13: 59: 14-$ 18

INFO [3568] connect. ConnectThread (282) $(3568) 10: 59: 14$ Seruerdata VPRRSER_Uo ice

INFO [2868] connect. ConnectThread $(339)(2868) 10=59: 14$ Serverdata VPARER_UI DEO

INFO [3072] connect.ConnectThread 423$)(3372) 16: 59: 14-$ INFO [3752] connect. ConnectThread $(563)(3752\rangle 10=59: 14-$

INFO [3972] connect. ConnectThread (442)(3872)10:59:14 -

INFO [3752] connect . Connect Thread $(394)(3752\rangle 13: 59: 14$ Seruerdata VARSER_PICTURE

INFO [3972] connect .ConnectThread $(563)(3872) 10: 59: 14$ -

INFO [3752] connect. ConnectThread $(394)(3752) 10: 59: 14-$ Seruerdata VPARSER_PICTURE

INFO [3672] connect . ConnectThread $(537\rangle\langle 3972\rangle 10: 59: 14$ 21101624

Figure 5

The interface for server-side users to download files 
ci d: WrqDisRadarServer VRelease VDisRadarServer. eze

INFO [2712] connect .ConnectThread(423)(2712)11:20:69 I NFO [2736] connect. Connect Thread (563)(2736)11:20:69

INFO [252] connect.ConnectThread(394)(252)11:20:69 Nrq Serverdata VARSER_PICTURE

INFO [2712] connect.Connect Thread(442)(2712)11:20: 39

INFO [3568] connect.ConnectThread(282)(3568)11:20:09 D: Mrq Serverdata \PRSER_Uoice

INFO [252] connect.ConnectThread(394)(252)11:20:B9 Wrq \erverdat a VPARER_PICTURE

INFO [2712] connect - Connect Thread $(563\rangle(2712) 11=20: 09$

INFO [2736] connect.ConnectThread(339)(2736)11:20:09 D: :rq $\backslash$ Serverdata $\backslash$ PARSER_UIDEO

INFO [252] connect.ConnectThread(394)(252)11:20:B9 Wrq $\backslash$ Serverdata \PARSER_PICTURE

INF0 [2712] connect. ConnectThead (537) (2712)11:20:09 20110921085645

INFO [252] connect.ConnectThread(394)(252)11:20:09 vrq Serverdata \PARSER_PICTURE

INF0 [252] connect. ConnectThread(394)(252)11:2日:B9 -

Figure 6

The page of file upload 

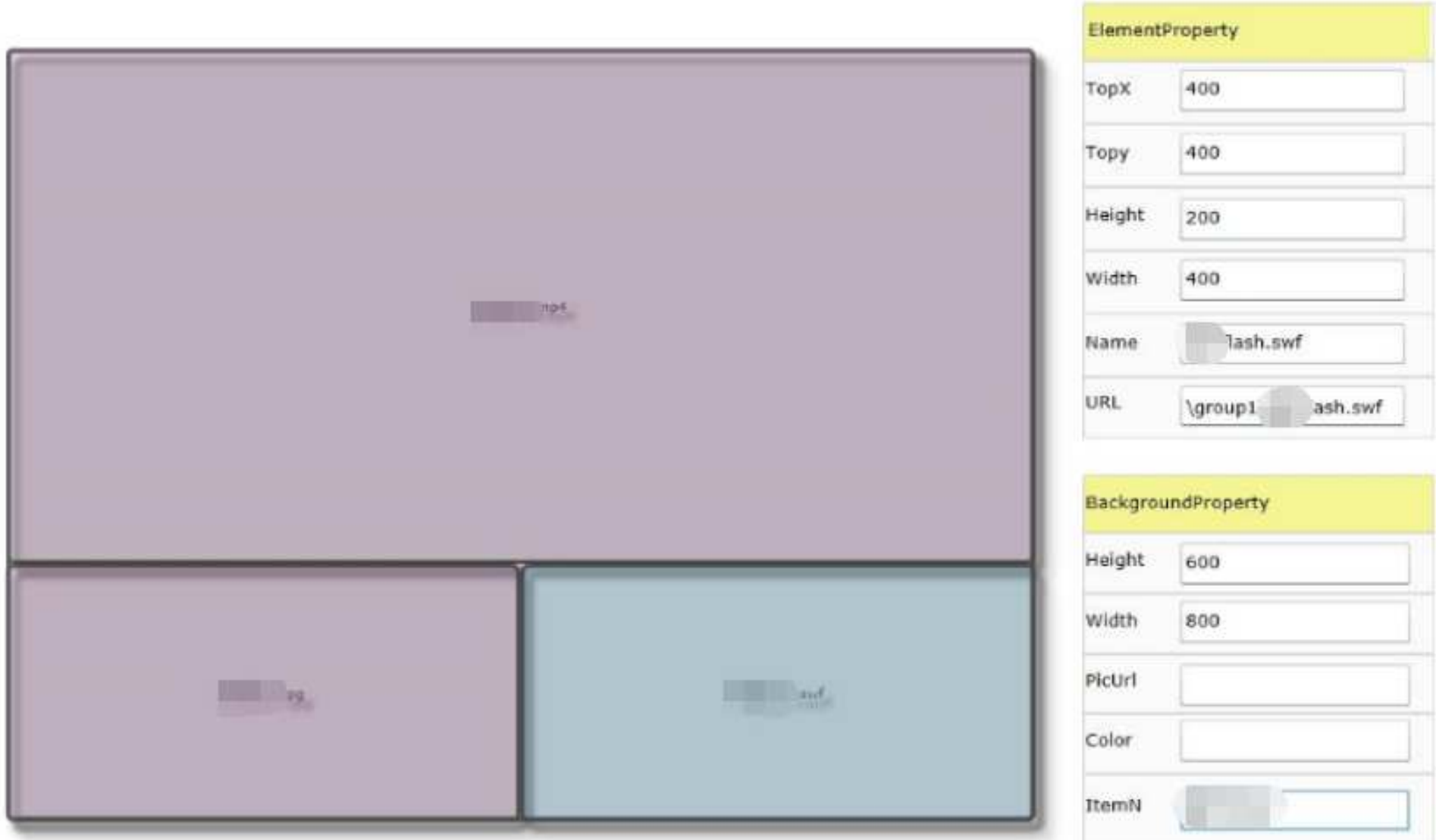

\section{BackgroundProperty}

\begin{tabular}{|l|l|}
\hline Height 600 \\
\hline Width 800 \\
\hline Picurl \\
\hline Color \\
\hline ItemN \\
\hline
\end{tabular}

Figure 7

The page of item arrange 

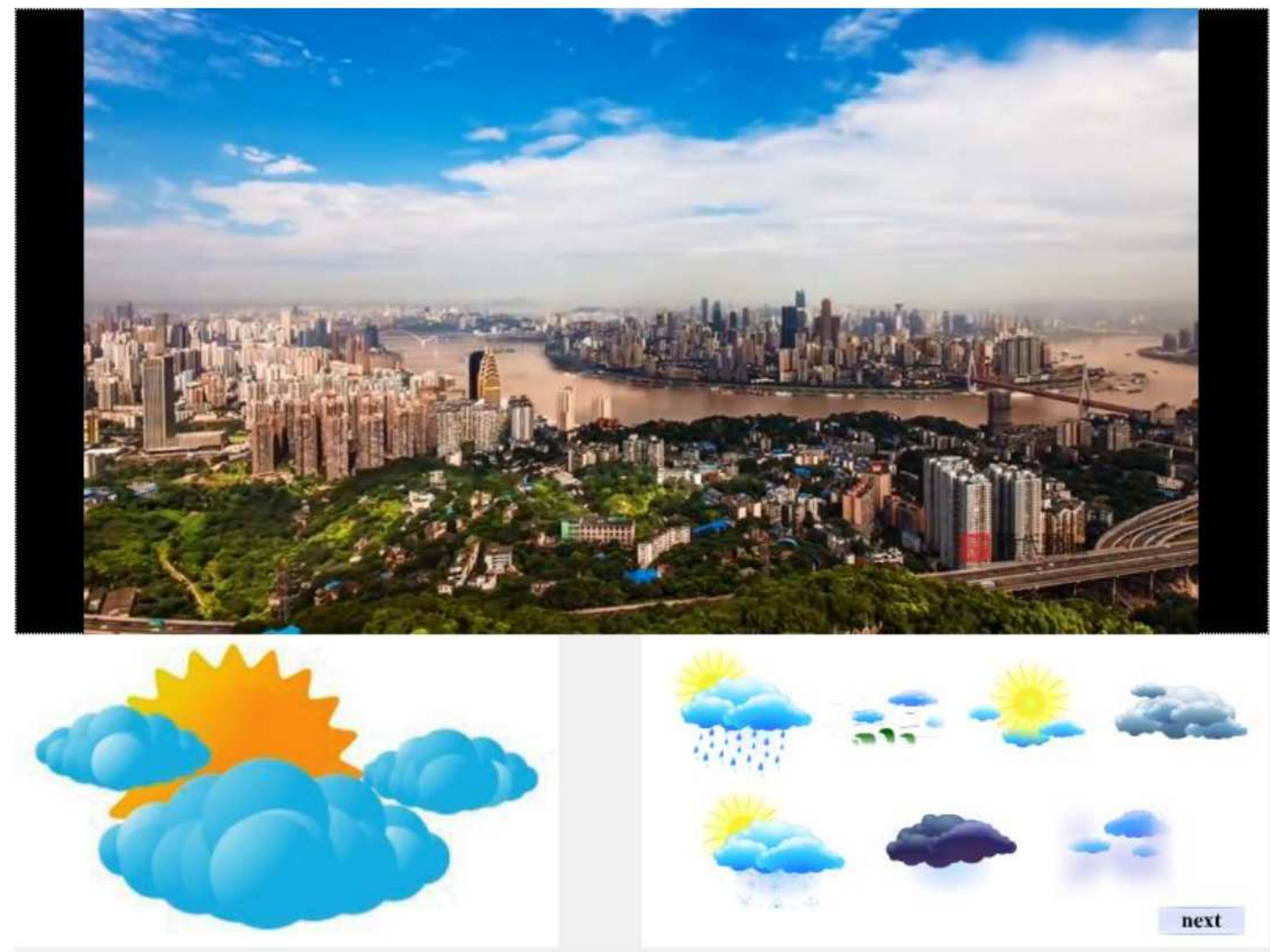

Figure 8

Play screen 